tion as after. Definite appendicitis is primarily a surgical disease, and if a special abdominal surgeon be available in case $I$ am attacked, I shall be operated on; if not I shall eliminante, starve, rest my intestinal canal, apply the abdominal bandage, but not the opium splint - except in the rarest of cases--and trust to God and the vis medicatrix Natura.

In brief, ideal surgery-if available--with 1 or 2 per cent. mortality is better than 20 or 25 per cent. mortality under medical or expectant treatment; but the latter is to be preferred to the chances of surgical interference at the hands of average general surgeons or practitioners. Under the above plan, if my patient dies I will feel as my old friend, the late Dr. Guhman, used to say, that "I had let him die honest."

Were I ten years younger I would, on account of my strong convictions on this question, go to some of the masters mentioned above and become a pupil for a year or more, if necessary, providing my hand could grasp their superb technic, that I might devote my remaining years to all sides of this question. As it is, I shall continue to study and observe these interesting problems in the hope that I may be helpful in the equally important realm of diagnosis as related to their elucidation, and I trust that my co-workers will bear with me if they deem my position as here presented too radical.

\section{OBSERVATIONS ON ASCH OPERATION FOR DEVIATION OF CARTILAGINOUS SEPTUM**}

BY MAX THORNER, $\dagger$ A.M., M.D.

Professor of Clinical Laryngology and Otology, Cincinnati College of Medicine and Surgery; Laryngologist and Aurist to the Cincinnati Hospital; Follow of the American Laryngological Association, and Member of the American Medical Association, etc. CINCINNATI, OHIO.

It is not so very long ago, not too long for the older ones among us to readily remember, that deviation of the septum was considered a veritable bugbear by the majority of rhinologists. The methods then in vogue to remedy this deformity were few and not generally satisfactory, so as to insure permanent results and relief of the train of symptoms produced by the more or less pronounced nasal stenosis. It is beyond the limits of this paper to enumerate all the different methods that have been devised, beginning with the contpressing forceps of Adams, or even with the procedures of the older surgeons, as Dieffenbach, Bolton, Gunn, Chassaignac and many others: nor do I propose to enter into any discussion of the etiology, pathology and symptomatology of this deformity and its local and general consequences for the patient. An immense progress, as far as the relief from stenotic and other conditions is concerned, which are produced by projections from the septum of whatever nature, was made when Bosworth, in 1886, devised his nasal saw. He opened up an entirely new field of nasal surgery by this procedure, and it looked as if all the difficulties resulting from anomalies of the septum had then come to an end. However helpful and masterly this device-simple as it was-proved to be, there remained still that large class of cases in which the triangular cartilage was bent toward the one side or the other, or sometimes in the shape of an $S$ toward both sides, causing one or more of the consequences of stenosis, which could not be relieved by sawing of a spur. These were the cases in which most of the other methods failed, for the reason that the natural resiliency of the

- Presented to the Section on Laryngology and Otology, at the Fiftietb Annual Meeting of the American Medical Assoclation, beld at Columbus. Ohio. Tune 6-9, 1899

$\dagger$ See obituary of the author, JouknaL, Sэpt. 2, 1899, p. 62? cartilage was not overcome. 'I'he other methods then in vogue can be divided into two groups, according to their general principle. They either attempted to simply push the curved cartilage by force into the normal position and keep it there by all kinds of devices, or they fenestrated the triangular cartilage at its most projecting portion by cutting a more or less extensive piece out of it, sometimes with preservation of the mucous membrane on one or both sides. It can not be denied that either of these methods may have been successful in certain cases, but in the majority the results were not permanent, for in principle both methods are wrong. The deviated cartilage has an enormous resiliency, which can not be overcome by simply bending it in the opposite direction and holding it there for a little while. Compare a piece of good strong watch-spring $(a, b, c$, Fig. 1), and have the two ends firmly fixed to unyield-

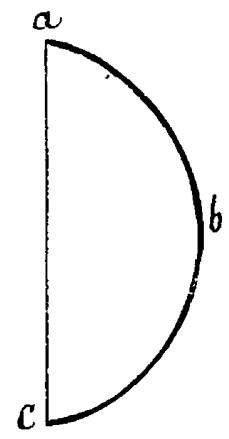

ing supports ( $a$ and $c$ ), the main piece forming a slightly curved arch $(a, b, c)$. Is it possible to press this spring into the straight line ( $a$ and $c$ ) and keep it there? This is not possible without breaking the spring, and thereby destroying its resiliency. And, furthermore, we all remember, from our first lesson in geometry, that between two points ( $a$ and $c$ ) the shortest is the straight line; all others are of necessity longer. Now, this applies directly to the subject under consideration. The resiliency of the cartilage must be first completely destroyed before it can be expected to be pushed into its proper place and be kept there, and this can only be done, as in the case of the watch-spring, by fracturing it and incidentally gaining sufficiently thereby to adjust the longer arch into the shorter straight line.

In my earlier days I have used the different methods one after anothes, and had finally adopted the method of Roberts, ${ }^{1}$ described in 188- It consists in the main in making a long incision with a curved bistoury along the crest of the deviation, supplementing it, if necessary. by additional incisions and then keeping the straightened septum in place by steel pins, which are introduced through the unobstructed nostril in front of the incision, then carried across it, and finally buried in the mucous membrane of the vomer. This method was more satisfactory to me than others, but was given up for the operation devised by Dr. M. J. Asch, when I became acquainted with it, because it has given me such uniformly good results and appeared to me so much in advance of every other method described at that time, that I have used it since to the exclusion of other methods. The method was originally described by Dr. Asch, ${ }^{2}$ and modified from time to time, as described by Dr. Emil Maver. ${ }^{3}-^{4}$ and the originator himself ${ }^{5}$. As this method has neither in this country nor abroad become sufficiently known to the profession, as is evidenced by the fact that even text-books that have only lately appeared do not make any mention of it, it may not be out of 
place to describe its technic as practiced at this time.

The instruments as devised by Asch and used at present are the following: A pair of scissors or cutting forceps. They are made in two sizes, the blades being at the distal end of two strong shanks, which curve outward from the lock and meet again in front. One blade is narrow and blunt, the other triangular and sharp. (Fig. 2.) Through this arrangement they act on the principle of the button-hole scissors. There is also made a pair of angular scissors (Fig. 3) which may be used to advantage in deviations that lie low down on the floor of the nose or in those requiring an incision downward. There are two strong compressing forceps (Fig. 4), with two long or short blades respectively. The blades have a perfectly smooth inner surface, are attached at an obtuse angle to the branches of the forceps, which form a curve between the lock and the blades proper, thus excluding any danger of crushing the membranous septum of the nose upon closure and

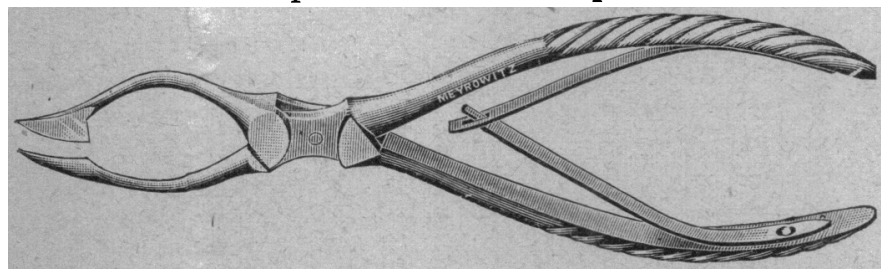

Fig. 2.-Asch's Straight Septum Scissors.

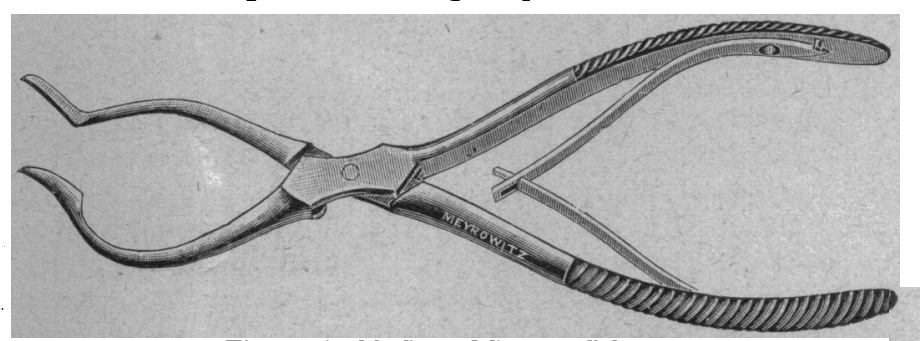

Fig. 3.-Asch's Curved Septum Scissors.

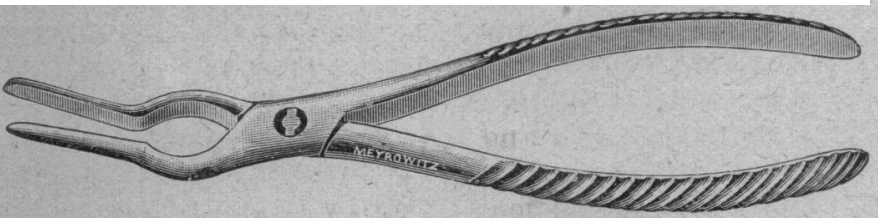

Fig 4.-Asch's Compressing Forceps.

also leaving a space of $1.5 \mathrm{~mm}$. between the closed blades, thereby preventing any. injury to the mucous membrane from too close approximation. There are also blunt and sharp separators (Figs. 5 and 6 ) to break up any adhesions that may exist between the deviation and the inferior or middle turbinate body and to detect the existence of obstructions posterior to the deviation, and to destroy them if this should be necessary. A set of six hollow vulcanite tubes, with perforated walls, completes the ontfit. 'The original tubes (Fig. 7) were somewhat smaller-only five to a set-and more rounded; the shape and size of the present tubes (Fig. 8), with more flattened walls, and six of them forming a set, were suggested by Dr. Emil Mayer and are generally known as the Mayer tubes. They are, as experience has shown, better adapted to the purpose for which they are used and are, as I understand, in general also preferred by Asch himself.

For the operation the patient is prepared in the usual manner, as it is ordinarily performed under general anesthesia. It is needless to say that all instruments, as well as the hands of the operator, must be carefully sterilized. Asch washes the nose out before operating, with an antiseptic spray. I personally omit this, as the nasal chambers beyond the vestibulum have been proved to be free of pathogenic germs in most eases; whereas, if they should contain them, the ordinary spray would not be possessed of any germicide action. However, I always have the skin of the face, and particularly of the nose, the upper lip and of the vestibulum thoroughly sterilized by scrubbing with soft soap, alcohol and 1-1000 solution of bichlorid of mercury, with the object of reducing the possibility of contact infection to a minimum, and with this end in view insist also on shaving, especially of a moustache, as it is impossible to thoroughly sterilize this nidus of infection carriers. The operation is usually done with direct light, but may also be performed with the reflected.

As soon as the patient is completely under the influence of the anesthetic the head is well drawn over the head of the table so as to prevent any blood from entering the larynx. The blunt separator is now introduced

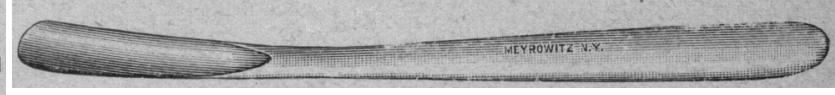

Fig, 5.-Asch's Blunt Separator.

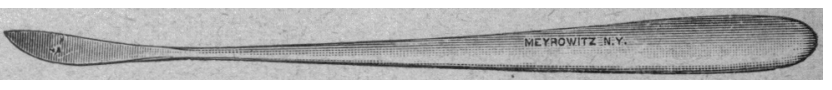

Fig. 6.-Asch's Sharp Separator.

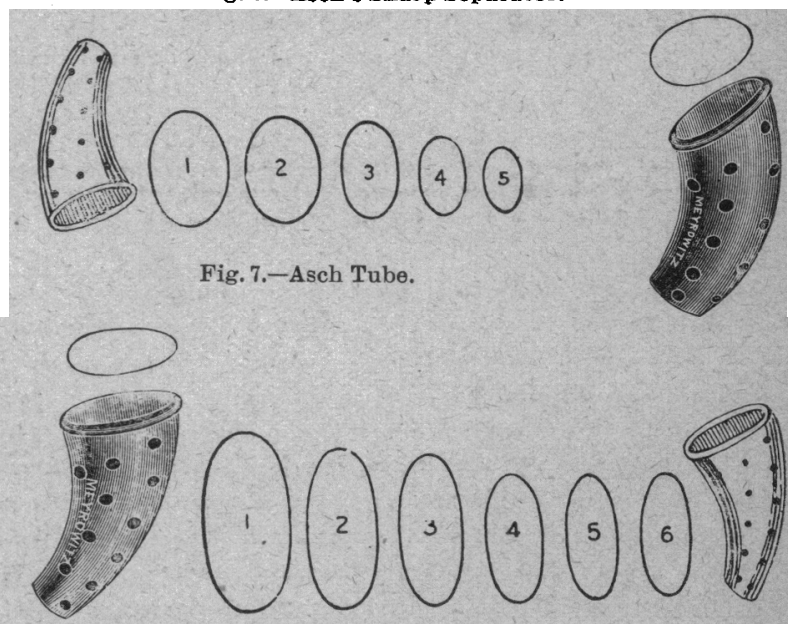

Fig. 8.-Mayer Tube.

into the narrowed nasal fossa, to sever any adhesions that may exist between septum and turbinates, and to discover the presence of posterior obstructions. If found they are opened with the sharp instrument, which is constructed after the fashion of a gouge. Should at this stage a brisk hemorrhage occur, which is however rare, it is readily controlled with an iced spray. The strong cutting scissors are now introduced parallel to the floor of the nose, the narrow blunt blade into the narrow side just over the line of greatest convexity, while the sharp blade within the concavity is just opposite the narrow edge, so that a plane drawn through their two edges would form a right angle with the plane of the septum. This is a very important rule. By now firmly compressing the handles of the instrument the blades are closed and the sharp one cuts through into the opposite side with a distinct and audible snap. The scissors are now completely withdrawn and reintroduced without delay, but in a vertical direction, and their blades pointing upward and as near as possible at a right angle with, and preferably just opposite to, the center of the line of the first incision. The second incision is then made by firmly closing the handles, leaving two incisions 
which intersect each other, and the instrument is then withdrawn from the nose. The four segments resulting from the crucial incision are now forcibly pushed across the median line into the concavity by the finger introduced into the narrow side, care being taken that they are thoroughly fractured at their base, as on this fracturing process depends the destruction of the resiliency of the deviated portion of the septum, and consequently the success of the operation. The compressing forceps is now introduced, one blade in each nostril, and the segments of the septum compressed by closing it firmly, thereby not only straightening the septum still further, but also causing the broken segments to overlap each other in the concavity. (b, Fig. 9.) By this process of overriding, the second condition of success, in addition to destroying the resiliency, is given to shorten the longer line of the deviated to the shorter one of the straight septum. (Fig. 9.) Thus the geometric axiom that the straight line between two points is the shortest, to which reference was made, is carried out.

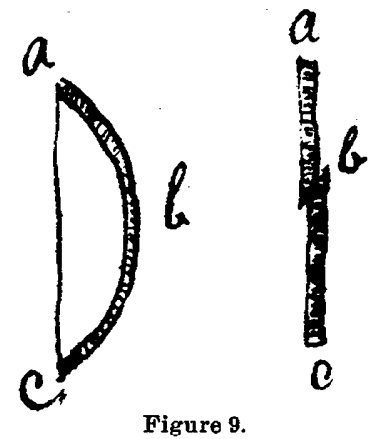

By this time the hemorrhage is usually quite brisk, but is controlled to some extent by the blades of the compressing forceps. It should be understood, however, that the forceps is simply meant as a compressing forceps, and any rolling, rocking or wabbling motion should entirely be avoided, as possibly inviting serious complications. If the segments are thoroughly broken at their base by the finger, the resiliency of the cartilage is completely destroyed, and it is not necessary to seize each one of the four segments separately with the forceps and to subject them to a twisting motion, sufficient to loosen its articulation, as recommended by H. H. Butts. ${ }^{8}$ After removal of the forceps, an iced antiseptic-or sterile-solution is sprayed in both nostrils, and a tube introduced into each side, the largest one that can be introduced into the previously stenosed side without exerting any force, and a smaller one into the other nostril, thus providing by even pressure, arrest of the hemorrhage and a support of the straightened septum. And it should here be stated that the vulcanite tubes are used exclusively as supports of the straightened septum until it has become fixed in its new position, and not to force it over to the other side. If this should become necessary the operation is not well done, and will probably be not entirely successful, and the tubes will be more or less productive of pain while they are worn.

After introduction of the tubes the operation is finished, the patient is placed in bed, ice-cold compresses are placed over his nose, and after his rallying from the anesthetic cold sprays are used every half hour. Twentyfour hours after the operation the tube from the wider side is removed and not replaced; the spray and cold applications are continued. Twenty-four hours later the tube on the other side is removed, the nostril thoroughly cleansed with sprays, and the tube, after thoroughly cleansing and sterilizing, is reintroduced. If this pro- cess is painful, cocain is applied in 4 per cent. solution, and if it should appear that the tube is difficult to introduce or cause pain, a smaller one is selected. One should always bear in mind that the tube should just be large enough to cause no discomfort, but to fit snugly. This tube must be removed and cleansed every day, the nose thoroughly sprayed or washed out with a warm sterilized solution; and the patient is usually allowed to leave the hospital on the third or fourth day. After this the patient usually learns to manipulate the removal and reinsertion of the tube himself, which becomes quite painless after one week, during which he should be daily seen by the surgeon; but it is usually sufficient after this for the surgeon to see the patient from one to three times a week, as the case may be, for the next four weeks, after which the tube may be finally withdrawn. The septum has now become sufficiently fixed in its new position not to longer need any support.

There are a few additional details which may be mentioned. While the secretions have an outlet through the hollow splints, it sometimes happens that they occasionally do accumulate in and behind the tube, and become thick and tenacious. I institute, therefore, in the early stages of the after-treatment, or add to the use of the spray that of the warm nasal douche, using any mild alkaline and antiseptic fluids, previously sterilized, under low pressure, the receptacle being held at the level of the head. I wish to emphasize that there is absolutely no danger to the middle ear from the nasal douche if properly carried out, $i$. e., with warm water sterilized solution, low pressure, not too much fluid at a time, head bent forward, mouth open, and the patient enjoined from swallowing while the fluid is in the nose. The relief given to the patients with the douche is very remarkable, and it also can be done while the tube is in situ. Before reinserting the tube I usually spray the nose with one of the liquid preparations of vaselin, containing menthol in 1 per cent. strength, and oil the tube with sterilized vaselin or olive-oil. Thus it is possible to render the patient quite comfortable at an early date. There is no doubt that granulation tissue will spring up in such a vascular organ as the nose, around, above and below the tube, sometimes even to some considerable extent. This must be treated in the usual manner and be removed either with the cold snare, or cauterized with the galvanocautery or any of the chemical caustics. This is the reason why the patient should present himself in regular intervals to the surgeon for the first five weeks after the operation, in order to enable him to be on the lookout for such occurrences. The position of the septum will not require any attention, as it will be permanently fixed, if the operation was properly done, and the tubular splints were well selested. That this latter is necessary stands to reason. The tubes-and this can not be repeated too often-are not to act as compressors, but as support; they hare the rôle of the splints, tubular though they happen to be. The conditions are so variable that one should not expect that one splint, even in different sizes, could answer all purposes, just as little as the surgeon could do with any other splint of a given shape for all cases of fracture of a certain description. Experience will soon teach that the size of the splint must be properly selected, that it sometimes must be slightly altered in shape, that for instance the anterior upper rim must be cut off so as to make it slanting, or again that it must be shortened posteriorly, etc. Sometimes it becomes necessary to support it with small wads of cotton, etc., but all these makeshifts are needed 
only in exceptional cases, and can not therefore militate against the usefulness of the splints in general. Experience, as in other things, will soon teach what to do. I wish to add that the splints as made at present can be sterilized in boiling water without changing their shape.

Regarding the portion of the septum where the free edges of the segments override each other and are liable to leave something thickening ( $b$, Fig. 9), as is to be expected from a consideration of the fact that a longer curve is reduced to a shorter straight line, it can be stated that these thickenings are generally very insignificant and will, if left to themselves, eventually disappear. However, if needed, they can be readily removed later on, and this may be done by electrolysis, galvanocautery, the knife curved on the fiat, the saw, the electro-trephine, etc., according to the conditions present and the preference of the operator. This applies also to spurs or posterior obstructions that may exist in addition to the deviation, and may require special consideration. AlI these additional operations should be done some time after the septum has been permanently fixed in its new position. There are but a few conditions which require preliminary operations. One of them is the luxation of the triangular cartilage from the columna. These are the only cases in which Emil Mayer ${ }^{7}$ operates in advance of the straightening of the septum by a submucous resection of the projecting piece of cartilage. There are, however, in my opinion, two more conditions which require preliminary operation. The first is the presence of nasal polypi in the concavity. If they exist in the other side also, they are probably behind the deviation and could not be reached until the stenosis is overcome. In one of my cases (No. 13) the concavity was packed with so-called mucous polypi, and it is self-evident that their removal had to precede the correction of the deformity of the septum. There is, however, another condition which was described by D. Bryson Delavan ${ }^{8}$ as a comparatively frequent occurrence. He found in eleven out of eighteen cases of deviation of the septum, a condition of hypertrophy of the middle turbinate bone in the otherwise spacious concavity, which was sufficiently large to interfere with the patency of the wider nasal fossa after the septum has been brought back to a vertical plane; or it may even interfere with the surgeon's efforts to push the septum back into the vertical plane. In such cases Roberts ${ }^{9}$ also demands the preliminary removal, either partial or total, of the turbinated bone, and from one experience which I had (Case 17), in which I do not do it, I am convinced it is the proper thing to do. This is an entirely different condition from that, which Mayer mentions, when he says, ${ }^{10}$ the hypertrophied "turbinated became so, because the volume of air-pressure was diminished in the (concave) cavity, and it was remarkable how small it became on the correction of the deformity." 'There certainly can not be any doubt about this, as it apparently refers to hypertrophy of the turbinate bodies; but the anomaly aforementioned is a hypertrophy or bullous enlargement of the turbinated bone or bones (Fig. 10) which will not yield or undergo a retrograde change after replacement of the septum, and which should receive attention before the operation on the septum, lest it produce a condition in the concavity which existed before the operation in the stenosed side. (Fig. 10.) It is, however, but just to mention that it is, at least in my experience, by no means a common occurrence.

As to the results obtained by this operation, sufficient time has elapsed since it was first performed, in 1882, by
Asch, and the number of cases operated on is large enough to arrive at definite conclusions. In 1898 Mayer was able to refer to 200 operations, which had been done from 1888 to $189 \%$, of which 78 eases had been operated on at the Manhattan Eye and Ear Hospital, and 122 at the New York Eye and Ear Infirmary. Of the latter the operations were distributed among the following operators: Asch, 39 operations; Mayer, 35 ; Hitchcock, 18; Adams, 16; Waterman, 3 ; Simpson, 3; McKernon, 2 ; Gage, 2; Whiting, Concannon, Ieonard, and Bardes, each 1. All of these cases, of which complete hospital records were kept, are recorded as cured. These 200 mentioned do not include any operated on in private practice. Since then a good many additional operations have been performed at the same institutions with equally good results. ${ }^{7}$ Butts ${ }^{11}$ mentions only three failures in 60 operations done at the Manhattan Eye and Ear Hospital, averaging less than one each for each member of the hospital staff who had tried the operation. Two of these failures were the result of not thoroughly carrying out the technic in their initial trials; the third by being compelled to abandon the after-treatment on account of an acute otitis media that manifested itself a few days after the operation, done in an undersized, poorly nourished Italian, 7 years of age. One year later, the boy having meanwhile been built up, the operation was repeated with complete success. Butts considers the operations as giving good and permanent results.

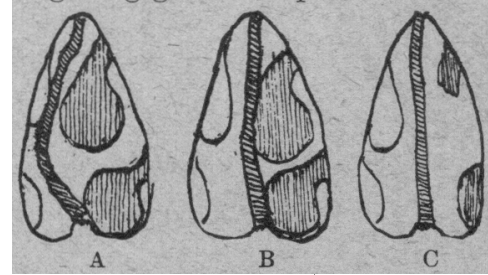

FIG. 10.-Diagrams showing deviation of septum with hypertrophy of middle turbinated bone, before and after operations : a, middle and inferior turbinated enlarged in the concavity; $b$, bad replacement, causing stenosis; c, correct replacement, after removal of turbinates. After Beaman Douglass.

My own personal experience is limited to 27 operations, done from 1892 to 1899 . With the exception of one partial failure during my early trials, when I had not yet fully mastered the technic from the scanty literature then extant, having never been present at any of these operations, all the others have been successful, and the cure obtained has remained so. There are but a few cases operated on less than a year ago, the shortest interval being, in one, about seven months. In all of these stenosis has been cured, the septum has remained in the median line, reflex symptoms, where they existed -in 2 cases-have been permanently relieved, and the correction of external deformity of the nose has been permanent. I wish to emphasize the fact that this operation has been, in my hands, signally successful for the relief of scoliosis of the nose, which in many instances is to the patient more distressing than the stenosis.

In three of my cases this deformity was very marked. The first case was that of a young student of theology, J. H., aged 18 years, who felt the disfiguration very keenly, inasmuch as it would be a great detriment to him in his appearance in the pulpit. The deviation was on the left side, obstructing the left nasal fossa entirely, and was of the most pronounced angular kind. The external nose was much distorted, the tip being turned toward the right and slightly upward. He was operated on Nov. 14, 1892, with the result of having the nasal stenosis relieved, and the deformity of the nose changed so minch for the better that when I saw him several years 
later, I was for a moment in doubt toward which side the nose had been bent.

Of my second patient, a girl 19 years of age, I have unfortunately lost my records. The cosmetic result was very good.

As I had the picture taken before operation only in my third case, I reproduce it here and refer to her a little more in extenso. Miss A. S., aged 16, came to the dispensary of my friend, Dr. S. E. Allen, principally for the purpose of having her nose straightened, as she said, and was referred by him to my hospital service for operation. On Dee. 13, 1898, she was operated on, Drs. Allen, Harris, Hines and the internes being present. As is my invariable custom in nasal operations, which are to be performed under an anesthetic, I applied a 10 per cent. solution of cocain, by means of a cotton carrier, to both sides of the septum, which was strongly deflected toward the right, the main convexity running from above backward to below forward. The whole dorsum

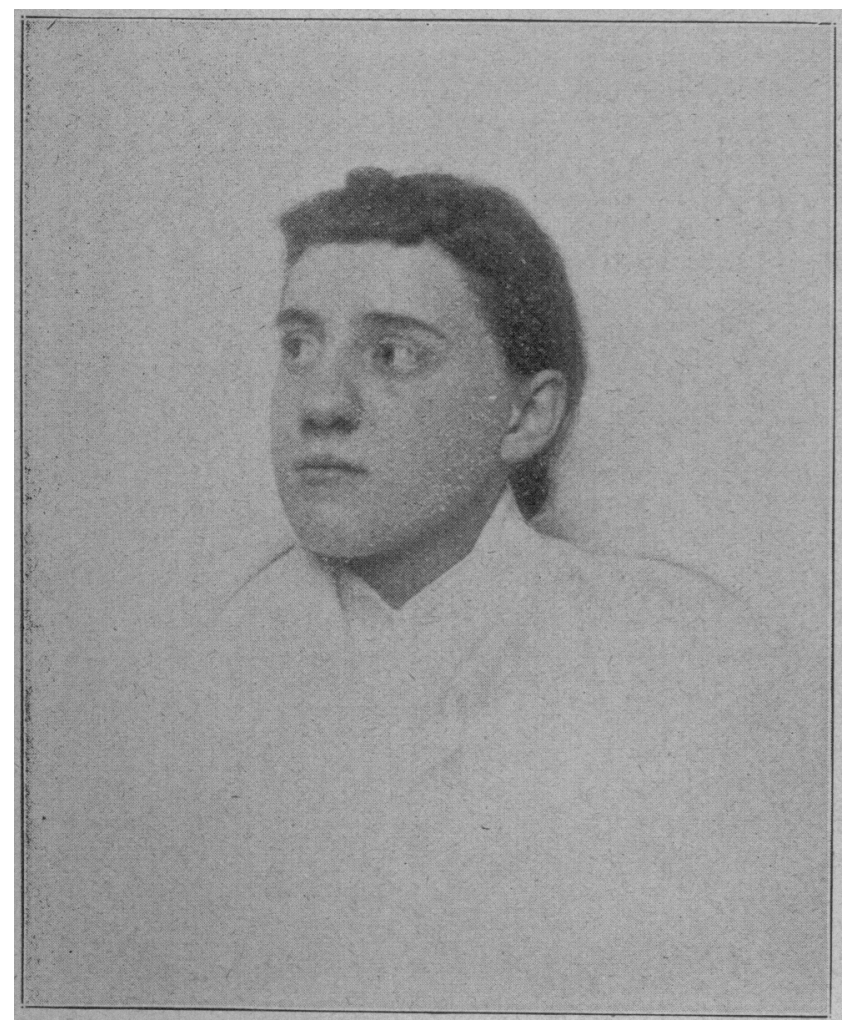

Fig. 11.-Taken before the operation.

nasi described a curve toward the left, having a kind of twist where the change from the sagittal line toward the left took place, with the tip of the nose pointing decidedly toward the left. (Fig. 11.) Chloroform anesthesia was used (Dr. Beson). The operation was done in the usual manner, as described above. After some adhesions had been broken up, and the resiliency of the septum thoroughly destroyed, it was found that after straightening the septum with the compressing forceps the tip of the nose could be readily brought into the median line. I then inserted the tubular splints, being very careful to select them of such size and shape as would not interfere with the cosmetic result, and bent thereupon the tip of the nose so much toward the right that the deformity was somewhat overcorrected, and held it in place by two long adhesive plaster strips of 1 inch in width, which were fastened one below and one in front of the left ear and extended across the face, exerting some considerable pressure on the tip of the nose, which had been previously protected by some absorbent cotton. These strips were left in situ for about one week, while the treatment of the interior of the nose was carried out as described. There were no complications except that later on some exuberant granulations within the nose required considerable attention. The result was as perfect as could be desired (Fig. 12, taken six months after operation), and, as she wrote to me but a few days ago, according to the assertions of her friends, she is not to be recognized as the same girl she was before the operation.

What now are the contraindications, disadvantages and dangers of this operation? I do not know of any other than those that would apply to any bloody operation in the nose done under an anesthetic. As to disadvantages and dangers, I could not mention any that are specific for this operation. While it is true that the hemorrhage is at one time, immediately after the crucial

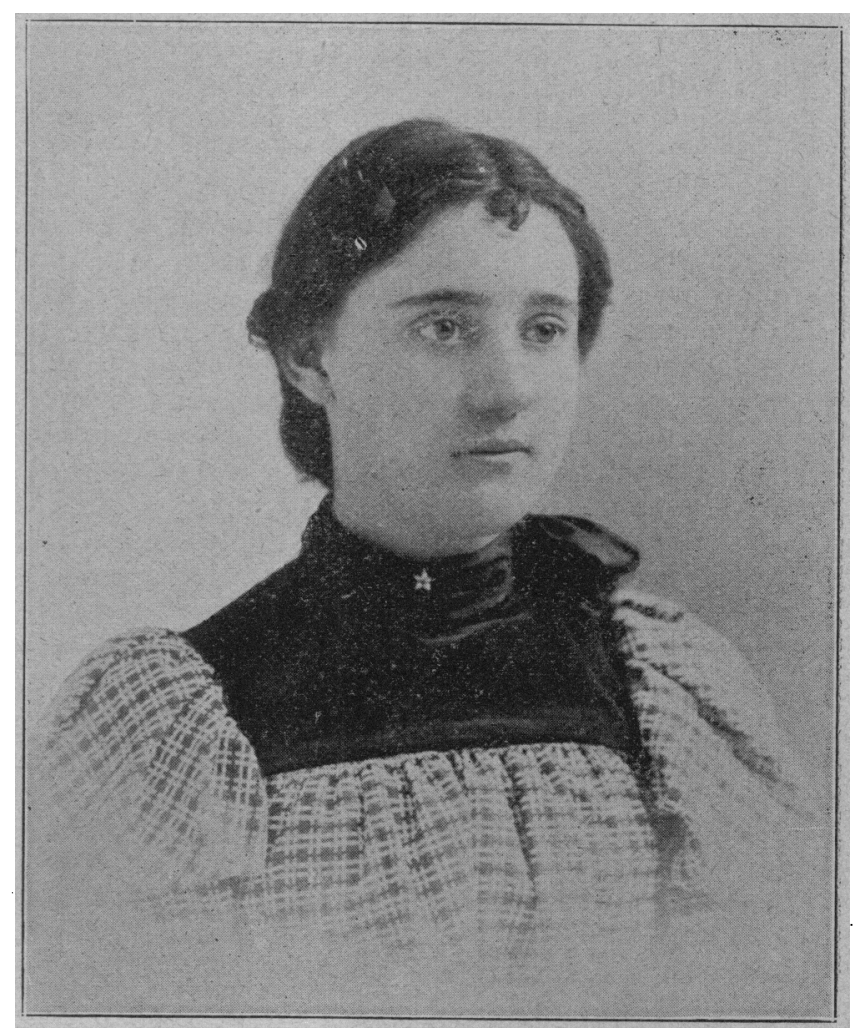

Fig. 12.-Taken after the operation.

incision has been made, decidedly brisk, it is not more so than in many other nasal operations, and it is readily controlled when the tubular splints are introduced. As to the danger of blood entering the larynx it must be remembered that the operation should be performed on the head lowered over the edge of the table; one might say the same regarding this danger in the operation for adenoid vegetations. Besides, the fact must not be lost sight of, inasmuch as the chloroform mask-or the ether cone - is removed at the beginning of the operation, that at this time the patient is generally not any more deeply under the influence of the anesthetic so as to abolish all reflexes, and that he is more than likely to swallow any blood that would happen to flow down into the pharynx. As to severe secondary hemorrhage, I have never seen nor heard of it. It is, however, possible that the application to the mucosa of the septum of the aqueous extract of the suprarenal capsule may greatly 
diminish the hemorrhage during the operation, as it has been reported to have done in other nasal operations. I have not had, so far, any opportunity to try it.

Stucky" speaks of "injury to inferior and middle turbinals" as a result of the Asch operation. From his description it does not appear whether it was done by the scissors, or by the tubes, in fact not whether it happened in the stenosed or in the wide side. But it must be remembered that in the stenosed side the turbinals are so small that they offer hardly any surface for being injured, and the blade introduced into the stenosed side is blunt. As to the wide side I can not see how the scissors, with some care, should injure the turbinals; but if these should be of an enormous size, they should be reduced, as stated above, by a preliminary operation. On the other hand, it is not easy to understand how the tubular splints could fracture the turbinals after the reposition of the septum, as all forcethis is essential and has been reiterated again and again - should be strictly avoided during the introduction. I must therefore conclude that the injury which Dr. Stucky saw in some patients who had been operated on by some other surgeon, must have been due to other causes, or were possibly the handiwork of some one who was in his first attempts at doing this work, and was possibly not sufficiently familiar with the technic and after-treatment.

Robert Levy ${ }^{13}$ reports a case in which, thirteen days after this operation had been performed, death occurred, in a rather poorly nourished individual who had been a sufferer from rheumatism and cardiac distress, and who had been addicted to the use of morphin. The cause of death was general septicemia, thrombus of the posterior cerebral artery, and cerebral softening. The thrombus was evidently due to syphilitic arteritis. 'This is plainly one of those accidents which are just as unfortunate as they are unavoidable, and which might have as well occurred after any other oneration on the septum, or for that matter on the turbinals also.

It must also be stated that, as a rule, no rise of temperature occurs after the operation; that the tubular splints, if kept clean, allow the patient to breathe through them, in contradistinction to the solid splints. However, I think Richardson ${ }^{14}$ is right when he says that in general there is some annoyance present as long as the tube or splint is worn. This applies naturally to any kind of splint or tube, as they are foreign bodies and must of necessity annoy the patient to some extent. Says he: "Nor can the fact be denied that such a body as a splint of hard rubber placed within the nasal eavity will produce temperature and inflammatory reaction. About the edges of such a foreign body, especially when placed in such a vascular organ as the nasal cavity, we are apt to, and do have exuberant growth of granulation tissue in proportion to the length of time the foreign body is worn." This would apply to all kinds of splints, and not to these alone. Yet, the after-treatment is, in reality, not painful after the first few days, during which one can readily overcome the pain by the use of some cocain; and the patient can really be made comfortable if a little care and judgment be exercised in the size and shape of the tubes. $A$ little mechanical skill will often adjust or shape a tube so that it will be absolutely non-troublesome, whereas it might otherwise annoy the wearer very much. In fact the patients very early learn to take the tubes out, clean them and reinsert them. In order to obviate these frequent changes and save the patient annoyance, Richardson proposes not to remove the prim- ary splint nor to change it, until its final removal, which could be done about the tenth day after the operation. He reports that he has done this successfully, finding that the septum is sufficiently well fixed in its new position after ten days, so as not to need support any longer. This proposition certainly deserves some consideration.

As to the anesthetic used, I wish to say that in my 27 operations 1 have done it twice with cocain applications alone, once under bronid of ethyl anesthesia, and 24 times under chloroform. This proportion shows that I do not think either cocain or bromid of ethyl the proper anesthetic to use for this operation. Whether one should use ether or chloroform is a matter of personal preference, as in other operations.

In conclusion, I am of the opinion that Asch has well merited the thanks of his confrères in devising and perfecting this operation. It has been said that the principle of cutting through the cartilage in order to overcome the resiliency was not new. What does this matter? The late Joseph O'Dwyer was the one who introduced his perfected method of intubation, and to him all the well-earned credit was given, although Bouchut had many years before made use of the same principle of introducing stationary tubes into the larynx. For that matter, the principle of crucial incisions was described by Réthi ${ }^{15}$ of Vienna, in the same year, 1890, when Asch announced his method. But what a difference between the two procedures? Réthi first makes crucial incisions into the mucous membrane of the concave side, with a special lancet-shaped knife, then pushes the mucous membrane back with a raspatory; then with a peculiarlyconstructed chisel, the septum is cut into four segments; after this the stenosed side is once more cocainized, two blunt retractors are introduced into the stenosed nostril and after measuring the distance of the crucial incision from the tip of the nose, a cut with a narrow tenotomy knife introduced into the narrow side is made in the prolongation of the primary incision, penetrating into the concave side. After this the septum is thoroughly weakened, and is pushed with a spatula into the median line. The septum is kept in its new position by oval rulcanized rubber tubes, which have been wrapped in iodoform or sublimate gauze, and which are placed in both nasal fosse. Réthi himself mentions his operation as "rather tedious"16 while the Asch operation can be readily done by any operator who has the least manipulative skill and some experience, in five minutes.

Of the greatest helps are the special instruments devised for this operation. It is true that one can make the crucial incisions with a bistoury, without needing any especially constructed pair of scissors. So it is possible to perform tonsillotomy with a scalpel, dispensing with the use of a tonsillotome. The compressing forceps is, moreover, a very handy instrument that should recommend itself to every surgeon. It differs very materially from the old Adams forceps, as any one can see, who will compare them, and has the great advantage of permitting its use in the posterior portion of the nose without endangering the columna. "It served me to an excellent purpose in the case of a lad, about 14 years of age, who was brought to my service at the Cincinnati Hospital with a fractured septum, the result of a basehall accident. 'The nose was considerably flattened, and the cartilaginous septum was almost reduced to a mass of unstable and crushed fragments. In narcosis, I introduced the compressing forceps, brought, as well as I possibly could by slow and even pressure, the fragments into juxtaposition, also lifted the dorsum 
nasi by this procedure, and kept them there by two snugly fitting Mayer tubes. In this instance I did, however, not remove the tubes until the sixth day, as I feared the badly crushed septum might not have enough stability to remain in position without support. After the tenth day the boy could be discharged with a moderately straight septum and a satisfactory cosmetic result. This case is not included in the number of operations done for deviation of the cartilaginous septum.

From all these considerations, from the experience of others, who have done this operation many times with universally good results, and from my own personal experience, extending over a period of over seven years. I am bound to consider this operation one of the best ever devised for deflection of the cartilaginous septum, no longer a bugbear to the rhinologist. It is simple, effective, and can be done in a short time, and the results are uniformly good and permanent, as has been sufficiently proved by the large number of cases operated on by various operators, and the time that has elapsed since the operations were performed. I can well imagine that cases may occur in which this operation is not applicable; but I have not as yet seen them. Nor do I wish to be understood as denying that other operations devised for the same purpose may have great merit, nor be productive of good results. But inasmuch as the Asch operation has been so very satisfactory in all my cases, I have never felt the necessity nor even the desire to try any other method. And I am absolutely convinced that among the many operative procedures devised by American rhinologists, this method will easily rank among those which mark a distinct progress in our specialty. BIBLIOGRAFHY.

1. Roberts: Phila. Polyclinic; Oct. 15, 1884. 1890.

2. Trans. of 12th Annual Meeting of the Am. Laryngol. Assn.

3. "Deviation of the Cartilaginous Septum, Its Cure." N. Y Med. Jour., Dec. 14, 1895 .

4. "Asch Operation for Deviation of the Cartilaginous Nasal Septum." Med. Record, Feb. 5, 1898.

5. Laryngoscope, 1899 , Vol. vi, p. 340.

6. Manhattan Tye and Ear Hospital Reports, Vol. iv, Jan., 1897, p. 78 .

7. Private commurication. Nio. 3.

8. Delaran, D. Bryson: Archives of Iaryngol., 1882, Vol. iii

9. Roberts, John B.: "Cure of Crooked and Otherwise De-

10. Laryngoscope, 1899 , vol. vi, p. 370.

11. Butts, H. Hoyle: Manhattan Fye and Ear Hospital Reports, ol. iv, 1897, p. 76.

12. Laryngoscope, Vol. $\nabla, 1898$, p. 147.

13. Levy, Rob: "Serious Consequences Following Intranasal Operations." Annals of Otol., Rhin., and Iaryngol,, Vol. vif, May, 1898, p. 241.

14. "The After-Treatment of Restored Deflected Nasal Septums." Annals of Otol., Rhin .. and Iaryngol., Vol. vii, Aug., 1898, p. 461.

Abdv. aus Wiener Klin. Woch., 1890, p. 23.

16. Ibid., p. 29

\section{LAMINECTOMY*}

BY JOHN C. MUNRO, M.D.

Assistant Visiting Surgeon, Boston City Hospital; Instructor in Surgery, Harvard Medical School. BOSTON.

The following cases of laminectomy, eighteen operations on seventeen patients are reported in the hope that they may furnish some encouragement for interference in one of the most interesting if discouraging fields for surgical operation, and because the writer is convinced that by a simplification of the technique the operation risk is much lessened, and that in the midst of numerous failures, so far as the power of giving life is concerner, nccasionally a brilliant success is possible,

* Presented to the Section on Surgery and Anatomy, at the Fiftieth Annual Meeting of the American Medical Association, held it Columbus, Ohio, June 6-9, 1899. while in certain other conditions, relief of suffering and prolongation of life is sure to follow. Furthermore, instead of the field for operation becoming narrower, there is every reason to believe that new and untried conditions may be amenable to surgical interference.

For most complete and invaluable neurologic data, the writer is indebted, in twelve of the cases, to Dr. William N. Bullard, whose interest and judgment have ever been most helpful and encouraging.

To begin with the most discouraging class, I will report three acute traumatic cases in which the lesion involved the cervical region.

CASE 1.-Edward A. W., 35 years old, after a fall down stairs on October 31, was brought to the Boston City Hospital; Dr. G. W. Gay's service. He was conscious on admission, with nearly total paralysis of the upper extremities, and total paraplegia, paralysis of the bladder sphincter, knee-jerks active and equal, sensation much diminished everywhere below the level of the third ribs and diminished below the clavicles. There was hyperesthesia of the neck, with apparent diminished sensation over both sides of the face, some tenderness over the third, fourth and fifth dorsal spines, marked tenderness from the seventh cervical upward, and anesthesia of both upper extremities, involving the whole of the right deltoid. Respiration was abdominal, with a good pulse.

At operation, six hours after injury, the seventh, sixth, fifth, and probably part of the fourth cervical laminæ were removed. There was no fracture, no extra or intradural hemorrhage. 'The dura was very tense and darker in color than normal. On incision, a clear serum spurted under much pressure. No evidence existed of any pressure on the cord, so far as could be told by exploring with a probe. Pulsation, which was absent before evacuation of the fluid, returned after loss of a considerable quantity. The cord felt normal to the touch. The dura was not closed. The external wound was closed with deep silkworm gut sutures, with rubber tissue drain.

The patient, at the close of operation, was in as good condition as at the start. On the next day he was doing well, with no change in the anesthesia, and on the second day he was better; could move his arms voluntarily, and there was a doubtful gain in sensation. Toward evening the temperature rose suddenly, followed by delirium and death.

Autopsy showed that the cord was soft at about the level of the second vertebra, about one inch above the opening made at operation. There was no sign of fracture nor dislocation.

CASE 2-Jesse L. P., $5^{r}$ years old, also entered on Dr. Gay's service. He had been caught in a shafting and carried around under a large fly-wheel. Probably his back was struck by the edge of the wheel, which at the same time forced him down underneath itself, where he lay with his feet up and curved around the wheel for some little time. At entrance he was in too much shock for operation. Stimulation was resorted to.

Operation was performed ten hours after injury, under ether. The patient had rallied considerably, and there was anesthesia below the second intercostal space. Incision was made over the lower cervical spine. The muscles about the sixth spinous process, especially on the left, were pulpified, and the process itself loose and pushed to the right. The seventh, sixth and fifth laminae were removed. There was apparently no pressure. The dura appeared normal. A small opening was 